\title{
Análisis de la motivación en las clases de Educación física de primer ciclo de Primaria
}

\author{
Ismael Varela-García1, Gema Paramio-Pérez² y Bartolomé J. \\ Almagro $^{3}$ \\ 1 Graduado en Educación Primaria, Mención EF (Universidad de Huelva) \\ 2 Universidad de Cádiz \\ 3 Universidad de Huelva \\ Email: ${ }^{2}$ gema.paramio@uca.es
}

\begin{abstract}
RESUMEN: La motivación en la enseñanza durante la Educación Primaria parece cada vez más necesaria e importante. Por esta razón, el objetivo de este trabajo fue analizar la motivación en clases de Educación Física de primer ciclo de Primaria. Para ello, se realizaron una serie de entrevistas, tanto a docentes $(n=2)$ como discentes $(n=8)$, de un centro escolar en Huelva. Tras realizar el análisis de dichas entrevistas, se obtuvo que los docentes de Educación Física le dan mucha importancia a la motivación dentro de sus clases, pero también destacan que al profesorado no se le forma académicamente como debería en este aspecto. Los docentes también señalan que la motivación en las clases de Educación Física es muy elevada, y los discentes lo corroboran en las entrevistas realizadas. Es por ello, que el principal objetivo del docente de Educación Física debe ser mantener la motivación en el desarrollo de sus clases. Por esta razón, se presentan una serie de estrategias prácticas para mantener o mejorar la motivación en las clases de Educación Física.
\end{abstract}

PALABRAS CLAVE: Educación, alumnado, metodología cualitativa, estrategias motivacionales, teoría de la autodeterminación.

\section{Analysis of motivation in Physical Education classes in the first cycle of Primary}

ABSTRACT: The motivation in teaching during Primary Education seems increasingly necessary and important. For this reason, the goal of this study was to analyze the motivation in Physical Education classes in first cycle of Primary. For that, a series of interviews were made for the teachers $(n=2)$ and learners $(n=8)$ of a school in Huelva. After the analysis of these interviews, it was found that Physical Education teachers give much importance to the motivation within their classes, but also note that teachers are not formed academically as it should in this regard. Teachers also point out that motivation in Physical Education classes is very high, and learners corroborated it in interviews. For that, the main goal of Physical Education teachers should be to maintain motivation in the development of their classes. For this reason, a number of practical strategies are presented to maintain or improve motivation in Physical Education classes.

KEY WORDS: Education, students, qualitative methodology, motivational strategies, self-determination theory. 


\section{INTRODUCCIÓN}

Un problema importante en la sociedad actual es el sedentarismo, el cual puede conllevar sobrepeso, obesidad y enfermedades crónicas asociadas en los individuos. Esto puede deberse a que se ha incrementado la desvinculación de los jóvenes escolares con la actividad físico-deportiva en los últimos años, especialmente en edades tempranas (Cecchini, Fernández-Losa, González, Fernández, y Méndez, 2012). El estudio realizado por Chacón, Arufe, Cachón, Zagalaz, y Castro (2016) revela que, actualmente, las nuevas generaciones se inclinan más por el ocio digital que por el ocio deportivo, como venía siendo lo normal hasta la fecha. Hoy en día, "se calcula que más de un $70 \%$ de la población en los países desarrollados no realiza la suficiente actividad física como para mantener la salud y controlar el peso corporal" (Bermúdez, 2015, p.4).

Desde la escuela, se dispone de la posibilidad de enfrentar estos hechos tan desfavorables para la vida del niño/a. El docente, y en este caso, de la especialidad de Educación Física, puede lograr en sus clases que el alumnado se interese por el deporte y la actividad física, con el objetivo de que también lo practique en horario extraescolar. Con ello, se intentará conseguir que los discentes lleven una vida activa y saludable, tanto en la escuela como fuera de ella. En esta línea, Castañón, Rodríguez y Granero-Gallegos (2011, p. 18) afirman que las clases de Educación Física deben ser "una experiencia positiva, evitando el fracaso, estrés y desmotivación, prolongando de esta manera la implicación de los escolares en las actividades deportivas y en la clases de Educación Física".

Hoy en día, parece que los niños y niñas de nuestra sociedad son cada vez más sedentarios, dando más importancia a los elementos tecnológicos que a la propia movilidad y vida activa del individuo (Arribas, Arruza, González, y Telletxea, 2007). Es por ello, que resulta de vital importancia aumentar la motivación de los jóvenes para fomentar unos hábitos de vida más saludables. Los docentes de Educación Física tienen una gran responsabilidad y posibilidad de intervención en ese ámbito, ya que cuentan con una hora y media semanalmente, aproximadamente, para intentar favorecer esa actitud deportiva en el alumnado. Los discentes deben descubrir las ventajas que posee llevar una vida saludable, donde observen claramente los beneficios de realizar actividad física.

Para conocer estas conductas de actuación que realizan los discentes respecto a la actividad física, es necesario estudiar la motivación de los mismos. Con ello, se pretende entender las concepciones de los discentes, conociendo por qué prefieren realizar unas tareas motrices $u$ otras, al igual que sus factores, como nos indican Gutiérrez y Caus (2006).

La motivación es "aquella parcela de la intervención pedagógica que trata de forma consciente y preconcebida de activar en los alumnos las necesidades de acción motriz, de tal forma que engendre en ellos la voluntad de conseguir los objetivos propuestos" (Lagardera, 1989, p. 105).

Uno de los modelos motivacionales más empleados para entender la motivación en las clases de EF ha sido el de la teoría de la autodeterminación (e.g. Cox, Smith, y Williams, 2008; Ntoumanis, 2001; Ntoumanis y Standage, 2009). La teoría de la autodeterminación (TAD) es una teoría empírica del comportamiento humano y el desarrollo de la personalidad (Ryan y Deci, 2017) que se ha ido desarrollando durante los últimos 40 años (Deci y Ryan, 1985, 2000, 2008, 2012; Ryan y Deci, 2000, 2017). La TAD se ocupa particularmente de cómo los factores sociales y contextuales apoyan o impiden el éxito de las personas a través de la satisfacción de sus necesidades 
psicológicas básicas de competencia, relación y autonomía (Ryan y Deci, 2017). A su vez, la teoría establece que existen diferentes tipos de motivación (motivación intrínseca, motivación extrínseca y desmotivación) a lo largo de un continuo, que va desde una conducta más autónoma (o autodeterminada) a una conducta controlada (o no autodeterminada) (Deci y Ryan, 2000). En esta línea, la TAD establece que la satisfacción de las necesidades psicológicas básicas aumenta la motivación autodeterminada y el bienestar (Ryan y Deci, 2000) en diferentes contextos: educación, deporte, trabajo, etc.

La TAD es una macroteoría que actualmente está compuesta por seis minitoerías (para más información ver Ryan y Deci, 2017), una de estas mini-teorías es la de las necesidades básicas. Las necesidades psicológicas básicas fueron definidas por Deci y Ryan (2000, p. 229) como "nutrimentos psicológicos innatos que son esenciales para un continuo crecimiento psicológico, la integridad y el bienestar". Además, esta teoría asume que existen tres necesidades psicológicas básicas, innatas y universales, que son: la necesidad de autonomía, la necesidad de competencia y la necesidad de relación con los demás (Deci y Ryan, 1985, 2000; Ryan y Deci, 2000). La autonomía se refiere al deseo de organizar su propia experiencia y comportamiento (Deci y Ryan, 2000), a sentirse el origen de sus acciones. La competencia se refiere a nuestra necesidad básica de sentir eficacia o maestría en lo que hacemos (Ryan y Deci, 2017). La necesidad de relación comprende el deseo de sentirse conectados con los demás (Deci y Ryan, 2000), es decir, sentirse conectados con otros y ser un miembro importante de los grupos sociales a los que pertenecen (Ryan y Deci, 2017). En este sentido, en el contexto educativo, cuanto más satisfechas estén estas tres necesidades psicológicas, se encontrará una motivación más autodeterminada en el alumnado en las clases de EF (e.g., Franco y Coteron, 2017; McDonough y Crocker, 2007; Méndez-Giménez, Fernández-Río, y Cecchini-Estrada, 2013).

La teoría de la autodeterminación (Deci y Ryan, 2000; Ryan y Deci, 2000) dispone diferentes tipos de motivación a lo largo de un continuo, en función del nivel de autodeterminación de la conducta. Por lo que, en dicho continuo se pueden encontrar, de menor a mayor autodeterminación, la desmotivación, la motivación extrínseca (regulación externa, regulación introyectada, regulación identificada y regulación integrada) y la motivación intrínseca. En la desmotivación, a los alumnos les falta intención de actuar (Deci y Ryan, 2000), y por tanto, es probable que su participación en clases sea desorganizada y acompañada de sentimientos de frustración, apatía, etc. La motivación extrínseca está determinada por recompensas externas o evitar un castigo (Deci y Ryan, 2000), y según esta teoría puede variar bastante en su autonomía relativa. Así, un alumno podría participar en las clases de EF por presiones externas (regulación externa), por evitar sentimientos de culpabilidad (regulación introyectada), porque quiere mejorar sus habilidades deportivas (regulación identificada) o porque quiere mantener un estilo de vida saludable o ser una persona activa (regulación integrada). Aunque la regulación integrada no parece estar presente en los niños o jóvenes (Vallerand y Rousseau, 2001). Por último, un alumno motivado intrínsecamente participaría en las clases de EF por la satisfacción y el placer inherente de la propia actividad.

Los docentes deben tratar de que su alumnado experimente motivación intrínseca por encima de la extrínseca, concienciándoles de que la verdadera importancia de la educación reside en que ellos quieran aprender, y no en una calificación que se obtenga en una materia, debido a que esto último se basaría en factores externos. Los autores Beltrán y Bueno (2000, p. 228), defienden en su libro la idea de que "si el estudiante mantiene un propio deseo o ganas de aprender, este aprendizaje se vería más fortificado y facilitado que si tuviéramos que hacerlo desde fuera, como docentes, mediante el uso de amenazas o premios". Valenzuelo y Nieto 
(2008) también le dan mayor importancia a la motivación intrínseca, pero añaden que este tipo de motivación se puede generar en alumnos/as que no disponen de ella, mediante ciertas condiciones que faciliten su aparición.

Por otro lado, según Naranjo (2009) la desmotivación en el aprendizaje por parte del alumnado puede aparecer por dos motivos principalmente. El primer motivo, se basa en que los contenidos que se enseñan están muy por encima del alcance de los discentes, por lo que ellos mismos no se ven capaces de aprenderlos. El otro motivo es todo lo contrario, basado en que el docente propone una enseñanza que está por debajo del nivel del alumnado, por lo que los discentes no muestran interés en alcanzarlos porque son demasiado fáciles. Ambas situaciones descritas producen desmotivación en el alumnado, por lo que hay que tratar de evitarlas. Lo ideal sería que se plantearan situaciones de aprendizaje para el alumnado a modo de desafíos, acordes con las características y capacidades de dichos discentes. Estos desafíos deben despertar la curiosidad del alumnado, con el objetivo conseguir la atención y el interés de los mismos en el aprendizaje (Naranjo, 2009). Una muy buena manera de obtener ese interés del alumnado es tratando con contenidos innovadores para ellos. En esta línea, González-Cutre (2017a) afirma que es necesario crear escenarios que satisfagan óptimamente la necesidad de novedad del alumnado, logrando consecuencias positivas. De hecho, recientes estudios de este autor (González-Cutre, Sicilia, Sierra, Ferriz, y Hagger, 2016; González-Cutre y Sicilia, 2018) están mostrando la novedad como una posible necesidad psicológica más (junto a la autonomía, competencia y relación con los demás), aunque todavía queda mucho por analizar y demostrar para que pueda ser incluida en la TAD. En cualquier caso, la satisfacción de la necesidad de novedad parece predecir la motivación intrínseca de alumnado de EF (Fernández-Espínola, Almagro, y Tamayo, en prensa; GonzálezCutre y Sicilia, 2018).

Siguiendo la teoría de la autodeterminación, los docentes de EF (factores sociales) desempeñan un rol fundamental sobre la satisfacción/frustración de las necesidades psicológicas básicas (autonomía, competencia y relación) de los estudiantes, generando tipos de motivación más o menos autodeterminados, que se van a manifestar en una serie de consecuencias como el aprendizaje, el disfrute durante las clases y el deseo de seguir practicando actividad física fuera del centro (González-Cutre, 2017b).

Por estas razones mencionadas anteriormente, es muy importante mantener un buen clima motivacional en la clase, con el objetivo de hacer más ameno el proceso de enseñanza-aprendizaje. Como nos indica García-Calvo, Santos-Rosa, Jiménez y Cervelló (2005, p. 22), "el clima motivacional, supone todo el conjunto de señales sociales y contextuales a través de las cuales los agentes sociales relacionados, en este caso los profesores de Educación Física, definen las claves de éxito y fracaso". Dichos autores también indican que es muy importante la labor que realicen los agentes sociales que trabajen en la educación del niño, ya que de ellos dependerá en gran medida que los discentes tengan un comportamiento motivacional u otro. Es por ello, que es de vital importancia que los docentes tomen conciencia de este aspecto, ya que su actitud afectará en gran medida a la educación del alumnado. Estos autores también proponen una serie de estrategias para mejorar el clima motivacional en las clases de Educación Física. Estas estrategias se basan en diseñar tareas de aprendizaje basadas en la variedad, utilizar ejercicios y juegos cooperativos, evaluar las tareas centrándose en la superación personal, planear objetivos a corto, medio y largo plazo adaptados a la comprensión del discente, etc.

Además de la importancia de la figura del docente para incrementar el clima motivacional en el aula, existen otros factores que son muy influyentes para conseguir 
una motivación de calidad en EF. Otras formas de aumentar la motivación del alumnado, especialmente en el aspecto intrínseco, sería fomentar la elección del estudiante en las actividades a realizar y transmitir un feedback positivo (MorenoMurcia, Joseph, y Huéscar, 2013). De esta manera, el discente mejorará su predisposición a realizar la tarea, ya que en gran medida, ha sido elegida por él. Como nos indica Gutiérrez y López (2012), un apropiado clima motivacional de la clase así como una disciplina fundamentada en razones intrínsecas y responsables, favorecerán la predisposición de los alumnos hacia la práctica física tanto en el contexto escolar como fuera de él. Por tanto, las experiencias y la motivación experimentada en EF resultan fundamentales para que los escolares sean físicamente activos.

Por lo general, son más escasos los estudios (e.g. Cecchini, Fernández-Losa, González, y Cecchini, 2013; Dorado, Paramio-Pérez, y Almagro, 2016; Navarro-Patón, Rodríguez, y Eirín, 2016; Valero, Delgado, y Conde, 2009) que se centren en la motivación en las clases de Educación Física en Primaria, ya que la mayoría de ellos van enfocados a la Educación Secundaria Obligatoria (e.g. Almagro, Navarro, Paramio, y Sáenz-López, 2015; Cera, Almagro, Conde, y Sáenz-López, 2015; González-Cutre y Sicilia, 2018; Méndez-Giménez et al., 2013; Moreno, GonzálezCutre, y Chillón, 2009; Sevil, Julián, Abarca-Sos, Aibar, y García-González, 2014; etc.), posiblemente porque es más factible que puedan rellenar los cuestionarios (por la madurez del alumnado de secundaria). Este trabajo trató de centrarse en una población poco estudiada, ya que se cree que es de vital importancia profundizar en la motivación del alumnado de primer ciclo de Primaria, debido a que es una edad idónea para inculcar unos valores saludables y físicos, que fomenten el desarrollo de una vida activa. En este sentido, el objetivo principal de este estudio fue analizar la motivación en las clases de Educación Física en $1^{\circ}$ y $2^{\circ}$ de Primaria.

\section{MÉTODO}

Para abordar el análisis de la motivación del alumnado de primer ciclo de Primaria se ha considerado como más efectiva el uso de la metodología cualitativa, por lo que como instrumento de investigación se empleó la entrevista.

\subsection{Participantes}

La muestra del estudio estuvo compuesta por un total de dos docentes de Educación Física y ocho alumnos de primer ciclo de primaria. Los docentes pertenecían al mismo centro escolar, e impartían clases a $1^{\circ}$ y $2^{\circ}$ de Primaria. En este sentido, el muestreo empleado fue intencional tanto para el alumnado como para el profesorado. A continuación, se presenta en la Tabla 1 las principales características de cada uno de ellos.

Tabla 1. Información de los docentes entrevistados

\begin{tabular}{|c|c|c|}
\hline \multicolumn{2}{|c|}{ Características del profesorado analizado } \\
\hline Variables & Docente 1 & Docente 2 \\
\hline Género & Femenino & Masculino \\
\hline Edad & 37 & 49 \\
\hline Años como docente & 15 & 26 \\
\hline
\end{tabular}


Además, en este estudio también se han entrevistado a ocho alumnos/as de primer ciclo, seleccionados según el curso al que pertenecían y al género. En la Tabla 2 se presentan las principales características de dicho alumnado, el cual pertenece al mismo centro escolar (centro público de Huelva).

Tabla 2. Información descriptiva de los discentes entrevistados

\begin{tabular}{|c|c|c|}
\hline \multicolumn{2}{|c|}{ Características del alumnado analizado } \\
\hline Discentes & Sexo & Curso al que pertenece \\
\hline Discente 1 & Masculino & $1^{\circ}$ de Primaria \\
\hline Discente 2 & Masculino & $1^{\circ}$ de Primaria \\
\hline Discente 3 & Femenino & $1^{\circ}$ de Primaria \\
\hline Discente 4 & Femenino & $1^{\circ}$ de Primaria \\
\hline Discente 5 & Masculino & $2^{\circ}$ de Primaria \\
\hline Discente 6 & Masculino & $2^{\circ}$ de Primaria \\
\hline Discente 7 & Femenino & $2^{\circ}$ de Primaria \\
\hline Discente 8 & Femenino & \\
\hline
\end{tabular}

\subsection{Instrumento}

Como instrumento para la recogida de datos se ha utilizado la entrevista semiestructurada. Se han utilizado dos guiones de entrevistas diferentes, dependiendo de si la entrevista era a un docente (ver Anexo 1) o a un discente (ver Anexo 2). La entrevista realizada a los dos docentes de Educación Física se componía de un total de 27 preguntas inicialmente, aunque dependiendo de la respuesta que daba se añadían algunas más, con el objetivo de profundizar en dicha cuestión. Para los discentes, la entrevista estaba compuesta por un total de 17 preguntas, adaptadas al nivel del alumnado. A estas cuestiones también se le podían añadir algunas más en función de la respuesta dada por el alumnado.

\subsection{Procedimiento}

Para llevar a cabo el proceso de entrevista, se realizó dos procesos diferentes, dependiendo del entrevistado. Para entrevistar a los docentes, se conversó con ellos para acordar una cita en alguna hora que tuvieran libre. Estas entrevistas duraron entre 15 y 20 minutos y se llevaron a cabo en el mismo centro escolar. En cambio, para entrevistar a los discentes de primer ciclo, se seleccionó a algunos de ellos con el objetivo de que fueran la mitad niños y la otra mitad niñas, y también la mitad de $1^{\circ}$ y la otra de $2^{\circ}$ de Primaria. Para poder entrevistarlos, se les pasó una autorización que debían traer firmada por los padres, donde se les explicaba en qué consistía la entrevista y el trabajo al que pertenecía. A estos ocho discentes se les entrevistó en un aula dentro del centro. Las entrevistas con el alumnado tuvieron una duración de entre 8 y 12 minutos, dependiendo de la extensión de las respuestas que daban los discentes.

\subsection{Análisis de los datos}


Para analizar los datos recogidos, lo que se hizo en primer lugar fue transcribir todas las entrevistas realizadas, tanto las 8 de los discentes como las 2 de los docentes. Una vez transcritas, se pasó a realizar el análisis de los datos recogidos. Para ello, se elaboró un documento con dimensiones, categorías y unos códigos (se le asignó tres letras a cada categoría y posteriormente un color) para ayudar a analizar y comparar la información. Se ha de decir, que dichos documentos varían según se analice al docente (ver Tabla 3) o al discente (ver Tabla 4), ya que el guion de entrevista fue diferente, así como la información que se podía conseguir de los docentes y del alumnado de esa edad (6-8 años). De este modo, empleando las tablas que se pueden observar a continuación para categorizar y codificar la información recogida en las entrevistas, se realizó un análisis del contenido del discurso.

Tabla 3. Dimensiones, categorías y códigos empleados para realizar el análisis de las entrevistas al docente

\begin{tabular}{|c|c|c|c|}
\hline Dimensiones & Categorías & Códigos & Descripción \\
\hline \multirow{2}{*}{$\begin{array}{c}\text { Datos } \\
\text { descriptivos }\end{array}$} & Datos & DAT & $\begin{array}{l}\text { Datos personales: edad, años de experiencia, centro } \\
\text { dónde trabaja, cursos a los que imparte clase... }\end{array}$ \\
\hline & Formación & FOR & $\begin{array}{l}\text { Formación que ha recibido en general y sobre } \\
\text { motivación. }\end{array}$ \\
\hline \multirow{2}{*}{ Ideología } & Conceptos & CNC & Concepto, tipos e importancia de la motivación. \\
\hline & Utilidad & UTI & $\begin{array}{l}\text { La importancia y utilidad que el profesor considera que } \\
\text { tiene la motivación en sus clases de EF. }\end{array}$ \\
\hline Docencia & Desarrollo & DES & $\begin{array}{l}\text { Cómo mantiene la motivación en el desarrollo de sus } \\
\text { clases. }\end{array}$ \\
\hline \multirow{3}{*}{ Contexto } & Conocimiento & CON & $\begin{array}{l}\text { El conocimiento que tiene sobre qué tipo de motivación } \\
\text { predomina en su alumnado. Conocimiento del } \\
\text { feedback y teoría de la autodeterminación. }\end{array}$ \\
\hline & Causas & CAU & $\begin{array}{l}\text { Cuáles son las causas, que a su parecer, pueden } \\
\text { provocar falta de motivación en los discentes. }\end{array}$ \\
\hline & Consecuencias & CNS & $\begin{array}{l}\text { Consecuencias que provocan la motivación o la } \\
\text { desmotivación en los discentes. }\end{array}$ \\
\hline Soluciones & Propuestas & PRO & $\begin{array}{l}\text { Propuestas que aporta el docente para mejorar la } \\
\text { motivación en las clases de EF. }\end{array}$ \\
\hline
\end{tabular}

Así, empleando el sistema de categorización presentada en las Tablas 3 y 4 , se analizaron los datos contenidos en las entrevistas transcritas. Para ello, se fue subrayando la información manualmente y asociándole un código (y un color). Esta codificación fue realizada por otro investigador de forma independiente y comparándose, para verificar la objetividad de los resultados.

Tabla 4. Dimensiones, categorías y códigos empleados para realizar el análisis de las entrevistas al discente

\begin{tabular}{|l|l|l|l|}
\hline Dimensiones & Categorías & Códigos & Descripción \\
\hline
\end{tabular}




\begin{tabular}{|c|l|l|l|}
\hline \multirow{2}{*}{$\begin{array}{c}\text { Datos } \\
\text { descriptivos }\end{array}$} & Datos & DAT & Datos personales: edad, centro dónde estudia. \\
\hline \multirow{2}{*}{ Docencia } & Utilidad & UTI & $\begin{array}{l}\text { La utilidad y beneficios que el discente considera que } \\
\text { tiene la EF. }\end{array}$ \\
\cline { 2 - 5 } & Diversión & DIV & Diversión del alumnado en las clases de EF. \\
\cline { 2 - 5 } & Desarrollo & DES & $\begin{array}{l}\text { Participación y feedback en el desarrollo de las clases de } \\
\text { EF. Análisis de la teoría de la autodeterminación: } \\
\text { autonomía, competencia y relación con los demás. }\end{array}$ \\
\hline \multirow{2}{*}{ Juegos } & Causas & CAU & $\begin{array}{l}\text { Cuáles son las causas, que a su parecer, provocan la } \\
\text { motivación o desmotivación en los discentes. }\end{array}$ \\
\cline { 2 - 5 } & Consecuencias & CNS & $\begin{array}{l}\text { Consecuencias que provoca la desmotivación en los } \\
\text { discentes. }\end{array}$ \\
\hline Soluciones & Propuestas & PRO & $\begin{array}{l}\text { Propuestas que aporta el discente para mejorar las } \\
\text { clases de EF. }\end{array}$ \\
\hline
\end{tabular}

\section{RESULTADOS}

Los resultados de este trabajo se van a organizar en función de las dimensiones y categorías establecidas previamente, que ayudaron a analizar dichos parámetros de una manera más exhaustiva. Se presentan en primer lugar los resultados obtenidos acerca del profesorado, y posteriormente, los del alumnado.

\subsection{Resultados de las entrevistas a los docentes de EF}

Analizando la primera dimensión datos descriptivos, en la categoría datos encontramos que el docente 1 tenía 37 años, y llevaba 15 ejerciendo como maestra, terminando su formación universitaria en el 99. En cambio, el docente 2, tiene 49 años, lleva 26 años como maestro, y acabó su formación universitaria inicial en el año 90. Ambos docentes ejercen EF en $1^{\circ}$ y $2^{\circ}$ de Primaria, respectivamente.

Respecto a la categoría de formación, destacar que ambos docentes han hecho la diplomatura de magisterio por la especialidad de EF. Además, se siguen formando, pero cada uno de distinta manera. El docente 1 se forma actualmente en cursos relacionados con el inglés, tutorías, orientación y necesidades educativas especiales. Por otra parte, el docente 2 señala que posee un centro de educación, y que por ello, aparte de los que recibe en el centro escolar, se forma constantemente. Respecto a la formación que han recibido sobre la motivación, se destaca que el docente 1 ha recibido muy poca, y que el docente 2 bastante, debido a que su centro escolar se fundamenta en la educación y motivación del alumno.

En la dimensión de ideología, y en la categoría de conceptos, ambos docentes definen de una manera muy correcta lo que entienden por motivación. En cambio, cuando 
se les pregunta por los tipos de motivación, el docente 2 sí que expone y ejemplifica varios tipos de motivación, pero el docente 1 da como respuesta "no conozco ningún tipo de motivación". Respecto a la utilidad y el conocimiento que supone la motivación en las clases de EF, destacar que ambos docentes creen que la motivación en las clases es indispensable para poder llevarla a cabo de la manera más correcta.

Respecto a la categoría desarrollo de la dimensión docencia, el docente 1 cree que para mantener la motivación del alumnado es necesario realizar actividades con objetivos que estén a su alcance. El docente 2, además de lo comentado anteriormente, añade que para mantener la motivación emplea su voz, decirles algo en público (si son cosas positivas) o en privado (si son aspectos negativos), pedirles colaboración para ayudar a los compañeros, etc. Los dos docentes desarrollan en sus clases los tres aspectos fundamentales de la teoría de la autodeterminación: autonomía, relación con los demás y competencia. Según los docentes, su alumnado es capaz de realizar los juegos y actividades que se le plantean, tienen una muy buena relación con los demás y son bastante autónomos. De este último aspecto, el docente 1 indica que "se pretende que (el alumnado) sea autónomo para que tomen la iniciativa en determinados momentos, al igual que para que sean responsables".

En la categoría de conocimiento, ambos docentes piensan que la motivación del alumnado es muy alta, y como destaca el docente 2, "(los profesores de EF) tenemos la suerte de que ellos ya vienen motivados, tienen una motivación innata acerca de esta asignatura y en estas edades". En la categoría de causas, encontramos que los docentes entrevistados piensan que esta motivación cambia a lo largo de la educación obligatoria, especialmente en las chicas. El docente 1 explica que "los niños/as de 5o y 6ㅇ les cuesta más trabajo aceptar juegos donde ellos no deciden a lo que quieren jugar". Por otro lado, el docente 2 se inclina porque estos discentes se especializan en un deporte extraescolar, y esto repercute en la EF escolar. Como consecuencias destacan que una buena motivación tiene consecuencias muy positivas, debido a que les facilita el aprendizaje, tanto motor como cognitivo (como se presenta en la Figura 1).

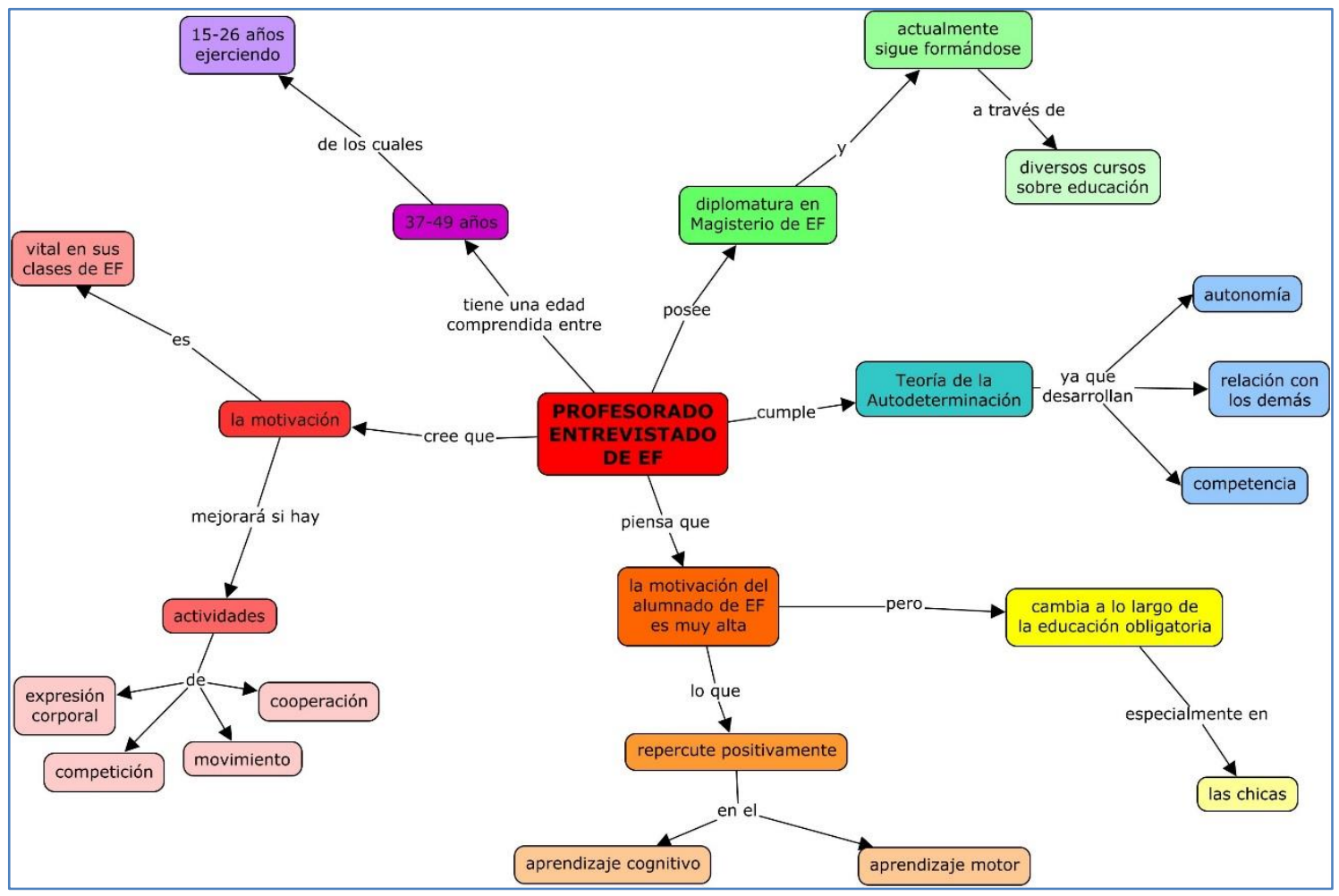


Figura 1. Mapa conceptual de las ideas más importantes del profesorado entrevistado

Por último, en el apartado de soluciones, el docente 1 destaca la formación del profesorado y su innovación. En cambio, el docente 2 se inclina más por la buena preparación previa de las sesiones a realizar, al igual que conocer al máximo el alumnado, especialmente sus necesidades motoras, para que ellos vean que la EF merece la pena hacerla. Respecto a las actividades que más le gusta a su alumnado, el docente 1 nombra especialmente a las relacionadas con la expresión corporal. En cambio, el docente 2 indica las tareas donde tienen que competir entre ellos, además de las actividades de movimientos, evitando las sedentarias y las de control. También señala que les encanta las de cooperación y las que se desarrollan en grandes espacios.

\subsection{Resultados de las entrevistas al alumnado de EF}

Respecto a la dimensión ideología, la mayoría responden afirmativamente a la pregunta de si es bueno hacer EF, con respuestas como la del discente 1 "es bueno porque es bueno para la salud", o como la del discente 4 "es bueno porque si no entrenamos el cuerpo, cuando haya algo que tengamos que correr porque haga falta, tu cuerpo va a estar flojo". En la categoría diversión, todos los discentes dicen que se divierten en clases de EF, debido a que están con sus amigos y realizan juegos en grupo.

En la dimensión docencia, la mayoría de sus concepciones sobre los docentes que les ha impartido EF son muy positivas, debido a que a todos les gustan las clases de EF que reciben, al igual que les gusta su docente, especialmente porque son buenos, cariñosos, alegres, pacientes, amables y razonables.

En la dimensión desarrollo, donde se le pregunta por si el docente les dice si lo están haciendo bien o mal, las respuestas suelen ser que sí que se les indica, tanto para bien como para mal. Respecto a cómo lo hace el docente, suele ser mediante diferentes feedbacks, acompañados ocasionalmente de demostraciones, como expresa en la entrevista el discente 2 "tienes que poner el pié aquí, luego allí, luego así... y de esta manera lo haces bien". En función a las preguntas relacionadas con la teoría de la autodeterminación (centrada en las necesidades psicológicas básicas), se comprobó que el alumnado se siente competente, debido a que es capaz de hacer las actividades que se proponen porque están adaptadas a su nivel. Por otra parte, la relación con los demás que posee el alumnado es muy buena, expresando en las entrevistas que tienen muchos amigos y se llevan bien con toda la clase. Por último, en el apartado de autonomía, destacar que la mayoría del alumnado piensa que el docente tiene en cuenta su opinión, especialmente en elegir a los compañeros con los que formar grupos, y no tanto en elegir los juegos.

En la dimensión juegos, los discentes exponen los juegos que más les gustan, que están íntimamente relacionados con los que realizan y, por lo tanto, con los que aprende con el docente en clases de EF. La mayoría del alumnado también expone que no hay ningún juego que no le guste, debido a que en todos se divierten y se lo pasan muy bien (aunque tiene sus preferencias).

Por último, en la dimensión de soluciones, cuando se les pregunta a los discentes que juegos harían si fueran maestros, la mayoría vuelve a repetir los juegos que más les gusta hacer en clases de EF, así como los que realiza en su tiempo libre. A continuación, en la Figura 2, se presenta un resumen de los resultados del análisis de las entrevistas al alumnado. 


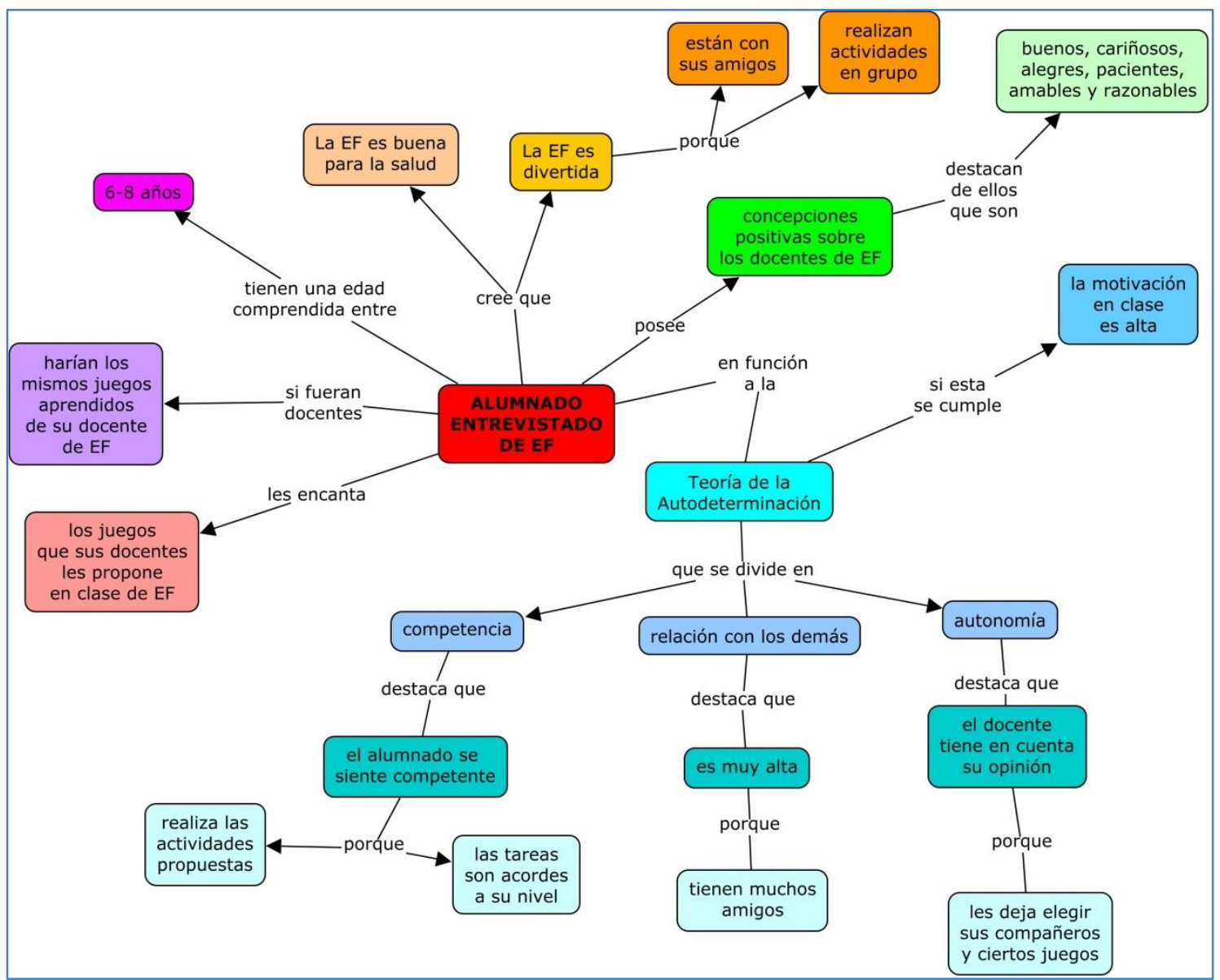

Figura 2. Mapa conceptual de las ideas más importantes del alumnado entrevistado

\section{DISCUSIÓN}

Del análisis realizado, en función de las respuestas dadas tanto por los docentes como los dicentes entrevistados, queda claro que ambas partes de la educación le dan mucha importancia a la motivación dentro del proceso de enseñanza-aprendizaje, al igual que en el estudio realizado por Castro, Piéron y González (2006). Estos autores también defienden que si el alumnado tiene una alta motivación, se mostrará más participativo en las actividades propuestas, al igual que hemos observado en las respuestas dadas por los entrevistados. De esta manera, se aprecia que el alumnado siente afecto por la EF y la relaciona con aspectos positivos.

Ambos docentes exponen en la entrevista que de la motivación depende el éxito en sus clases de EF, como indica Escudero (2009), aclarando que "todo profesor de Educación Física debe lograr que sus alumnos se encuentren motivados hacia las ejecuciones que se realizan, de ello depende en gran medida el éxito de la clase".

Respecto a los datos obtenidos de la entrevista, se puede destacar que los docentes no reciben apenas una formación relacionada con la motivación, sino que para aprender del tema deben de realizar cursos especializados en dicha materia. Además, echan en falta la necesidad de recibir formación sobre esta temática, porque consideran a la motivación como un elemento fundamental dentro de las clases de EF. Como expone Navarrete (2009), "cada alumno se motiva por razones diferentes". Debido a ello, se hace 
indispensable formarse sobre cómo mantener o mejorar la motivación hacia las clases de EF. Esto se podría conseguir formando al docente en sus estudios universitarios, y posteriormente, con cursos donde sigan aprendiendo sobre motivación.

Los docentes entrevistados, consciente o inconscientemente, siguen algunas premisas incluidas en la teoría de la autodeterminación de una manera correcta, debido a que tratan de satisfacer las tres necesidades psicológicas básicas del alumnado: autonomía, relación con los demás y competencia. Únicamente en el aspecto de autonomía no se ejerce totalmente, o al menos así lo percibe su alumnado, especialmente los de $1^{\circ}$ de Primaria, los cuales exponen que sí que se les deja autonomía para formar grupos con quien quiera, pero no se les permite elegir los juegos a realizar. Posiblemente, como indica Cecchini et al. (2013), con la metodología empleada, los contenidos trabajados, la forma de ofrecer feedback, etc. en las clases de $E F$, estos docentes han creado un clima de aula donde la motivación es muy elevada.

Debido a la motivación elevada que destacan los docentes de EF en su alumnado, ambos piensan que la función del docente en esta etapa educativa no es la de crear esa motivación, sino la de mantenerla. Es por ello, que se hace fundamental conocer estrategias para mantener esa motivación durante toda la clase de EF. Un aspecto a destacar es que ambos docentes se inclinan por utilizar materiales novedosos en sus clases, con el objetivo de aumentar o mantener la motivación, al igual que trabajar en grupo, adaptar los ejercicios al nivel del alumnado, participar con ellos en las actividades, reconocer los logros del alumnado, tener en cuenta su opinión, entre otros. La mayoría de estas estrategias de motivación también son defendidas por diferentes autores (Almagro et al., 2015; Escudero, 2009; González-Cutre, 2017b), con el objetivo de aumentar o mantener la motivación en EF de los discentes.

Los docentes entrevistados defienden la idea de que la motivación en las clases de EF disminuye a lo largo de la educación obligatoria, especialmente en las chicas. El docente 2 dice que no se innova demasiado en este aspecto, y que está seguro de que "si se propusieran actividades modernas como spinning, zumba, etc., las chicas estarían mucho más motivadas en las clases". Es probable, como apunta Moreno y Cervelló (2004), que las clases que se imparten en Educación Física sean menos entretenidas y motivantes en comparación con las actividades físico-deportivas que realizan los alumnados en su tiempo de ocio. Así mismo, estos autores también defienden que el alumnado que practica alguna actividad físico-deportiva en su tiempo de ocio valora más las clases de EF en el centro escolar. Por ello, se debe fomentar e intentar concienciar al alumnado para que practique actividades físico-deportivas en horario extraescolar, y buscar una relación entre estas actividades y las planteadas en el colegio, con el objetivo de aumentar la motivación en la participación de las mismas.

En cuanto a las limitaciones del trabajo, cabe señalar que la muestra utilizada consta de dos docentes y ocho discentes de un mismo centro, por lo que el análisis obtenido solo será válido para ese contexto; en este sentido, en un futuro sería interesante contar con más participantes de otros centros escolares para comparar los resultados obtenidos. Sin duda, son necesarias más investigaciones de este tipo, que permitan profundizar un poco más y entender mejor los procesos motivacionales en las clases de EF de Primaria.

Respeto a las posibles implicaciones prácticas, en función de lo analizado en el presente trabajo, se han propuesto 5 estrategias prácticas para aumentar la motivación en clases de EF con el alumnado de primer ciclo de Primaria:

- Hacer las tareas en grupos, con el objetivo de que el alumnado realice las actividades con los compañeros que desee (permitirles elegir algunas veces) 0 
con los que le toque (realizar agrupaciones de manera aleatoria), desarrollando así su relación con los demás.

- Adaptar las actividades al nivel del alumnado, con el objetivo de que aprendan habilidades de su zona de desarrollo próximo. Por lo tanto, evitar tareas muy sencillas o muy complejas, en función del nivel del alumnado.

- Dar autonomía al alumnado, especialmente en la elección de juegos o actividades, y también en la elección de compañeros para tareas cooperativas. También es importante para ello, escuchar e interesarse por la opinión del alumnado (teniendo siempre a actitud positiva ante lo que proponen 0 comentan los/as alumnos/as)

- Diseñar las sesiones teniendo en cuenta las preferencias y los gustos de los discentes, con el objetivo de aumentar el interés por las actividades que se proponen desde la EF.

- Introducir elementos novedosos en las sesiones, como pueden ser la utilización de nuevos materiales o dinámicas distintas, respecto al desarrollo de las clases que se daban hasta la fecha.

\section{CONCLUSIONES}

El objetivo principal de este trabajo fue analizar la motivación en las clases de Educación Física en primer ciclo de Primaria; en este sentido, cada docente entrevistado emplea una serie de recursos para mantener la motivación de sus discentes en las clases de EF, ya que ambos docentes creen que la motivación del alumnado por la asignatura es muy alta, por lo que la función del docente es mantenerla. Además, los resultados del análisis de contenido reflejan la escasa formación que recibe el docente de EF sobre motivación, la gran posibilidad de motivar al alumnado que poseen los docentes de EF y la importancia de emplear estrategias (en línea con la teoría de la autodeterminación) para conseguir una elevada motivación en clase. Algunas estrategias que pueden ayudar a mantener o mejorar la motivación en clases de EF con niños de entre 6-8 años serían: proponer tareas en grupos (con flexibilidad a la hora de organizar los grupos), adaptar las actividades al nivel del alumnado (evitando tareas muy sencillas o muy complejas), dar autonomía al alumnado (especialmente en la elección de juegos o actividades), diseñar las sesiones teniendo en cuenta las preferencias y los gustos de los discentes (para aumentar el interés por las actividades) e introducir elementos novedosos en las sesiones (e.g., utilización de nuevos materiales, juegos o dinámicas distintas...).

\section{REFERENCIAS}

Almagro, B. J., Navarro, I., Paramio, G., y Sáenz-López, P. (2015). Consecuencias de la motivación en las clases de educación física. Revista Digital de Educación Física EmásF, 24, 26-41. Recuperado de http://emasf.webcindario.com/ Consecuencias de la motivacion en las clases de EF.pdf

Arribas, S., Arruza, J. A., González, O., y Telletxea, S. (2007). Validación de una escala reducida de utilidad percibida de la práctica de la actividad física y el deporte. Revista Internacional de Ciencias del Deporte, 3(7), 34-48. Recuperado de http://www.cafyd.com/REVISTA/00704.pdf 
Beltrán, J., y Bueno, J. A. (1995). Psicología de la Educación. Barcelona: Editorial Boixareu Universitaria. Recuperado de https://books.google.es/ books? id=AwYlq11 wtjIC\&printsec=frontcover\&hl=es\#v=onepage \&q\&f=false

Bermúdez, C. (2015). Implicaciones educativas de los beneficios de la actividad físicodeportiva. E-motion. Revista de Educación, Motricidad e Investigación, 5, 3-16. Recuperado de http://www.uhu.es/publicaciones/ojs/index.php/e-motion/article/view/2638/2518

Castañón, I., Rodríguez, N., y Granero-Gallegos, A. (2011). Orientaciones de meta de los jóvenes escolares del colegio el buen pastor de Murcia. Espiral. Cuadernos del Profesorado, 4 (8), 13-21. Recuperado de http://www.cepcuevasolula.es/espiral/ articulos/ESPIRAL VOL 4 N 8 ART 2.pdf

Castro, M. J., Piéron, M., y González, M. A. (2006). Actitudes y motivación en Educación Física escolar. Retos, 10, 5-22. Recuperado de https://dialnet.unirioja.es/servlet/articulo?codigo=2089227

Cecchini, J. A., Fernández-Losa, J. L., González, C., y Cecchini, C. (2013). Aplicaciones del modelo de autodeterminación en la educación física de primaria. Revista Latinoamericana de Psicología, 45(1), 97-109. http://dx.doi.org/10.14349/rlp.v45i1.1317

Cecchini, J. A., Fernández-Losa, J. L., González, C., Fernández J., y Méndez, A. (2012). La caída de la motivación autodeterminada en jóvenes escolares. Revista EuroAmericana de Ciencias del Deporte, 1(1), 25-31. Recuperado de http://revistas.um.es/sportk/article/view/185531/153601

Cera, E., Almagro, B. J., Conde, C., y Sáenz-López, P. (2015). Inteligencia emocional y motivación en educación física en secundaria. Retos, 27, 8-13.

Chacón, R., Arufe, V., Cachón, J., Zagalaz, Maㅡ L., y Castro, D. (2016). Estudio relacional de la práctica deportiva en escolares según el género. Revista EuroAmericana de Ciencias del Deporte, 5(1), 85-92. Recuperado de http://revistas.um.es/sportk/ article/view/249161/189421

Cox, A. E., Smith, A. L., y Williams, L. (2008). Change in Physical Education motivation and physical activity behavior during middle school. Journal of Adolescent Health, 43(5), 506-513. https://doi.org/10.1016/j.jadohealth.2008.04.020

Deci, E. L., y Ryan, R. M. (1985). Intrinsic motivation and self-determination in human behavior. New York: Plenum.

Deci, E. L., y Ryan, R. M. (2000). The "what" and "why" of goal pursuits: Human needs and the self-determination of behaviour. Psychological Inquiry, 11(4), 227-268. doi: 10.1207/S15327965PLI1104_01

Deci, E. L., y Ryan, R. M. (2008). Self-determination theory: A macrotheory of human motivation, development, and health. Canadian Psychology, 48, 182-185.

Deci, E. L., y Ryan, R. M. (2012). Self-determination theory. En A. W. Kruglanski, P. A. M. Van Lange y E. T. Higgins (Eds.), Handbook of theories social psychology (Vol. 1, pp. 416-437). London: SAGE.

Dorado, E., Paramio-Pérez, G., y Almagro, B. J. (2016). Análisis de la motivación en las clases de Educación Física en Primaria. e-Motion. Revista de Educación, Motricidad e Investigación, 7, 3-12. http://dx.doi.org/10.33776/remo.v0i7.3136

Escudero, D. (2009). La motivación hacia las clases de Educación Física. Lecturas: Educación Física y Deportes, Revista Digital, 134. Recuperado de 
http://www.efdeportes.com/efd134/la-motivacion-hacia-las-clases-deeducacion-fisica.htm

Fernández-Espínola, C., Almagro, B. J., y Tamayo, J. A. (en prensa). Predicción de la intención de ser físicamente activo del alumnado de Educación Física: un modelo mediado por la necesidad de novedad. Retos, 37. Recuperado de https://recyt.fecyt.es/index.php/retos/article/view/70946

Franco, E., y Coterón, J. (2017). The effects of a physical education intervention to support the satisfaction of basic psychological needs on the motivation and intentions to be physically active. Journal of Human Kinetics, 59, 5-15.

García-Calvo, T., Santos Rosa, F. J., Jiménez, R., y Cervelló, E. M. (2005). El clima motivacional en las clases de Educación Física: una aproximación práctica desde la Teoría de Metas de Logro. Apunts. Educación Física y deporte, 81, 21-28. Recuperado http://www.raco.cat/index.php/ApuntsEFD/article/viewFile/ 300929/390375

González-Cutre, D. (2017a). ¿Qué papel juega la satisfacción de la necesidad de novedad en la motivación humana y cuál es su aplicación al ámbito de la actividad física y el deporte? e-Motion. Revista de Educación, Motricidad e Investigación, 9, 1-2. http://dx.doi.org/10.33776/remo.v0i9.3284

González-Cutre, D. (2017b). Estrategias didácticas y motivacionales en las clases de educación física desde la teoría de la autodeterminación. e-Motion. Revista de Educación, Motricidad e Investigación, 8, 44-62. https://doi.org/10.33776/remo.v0i8.3268

González-Cutre, D., y Sicilia, Á. (2018). The importance of novelty satisfaction for multiple positive outcomes in physical education. European Physical Education Review, 20(10), 1-17. doi: 10.1177/1356336X18783980

González-Cutre, D., Sicilia, Á., Sierra, A. C., Ferriz, R., y Hagger, M. S. (2016). Understanding the need for novelty from the perspective of self-determination theory. Personality and Individual Differences, 102, 159-169.

Gutiérrez, M., y Caus, N. (2006). Análisis de los motivos para la participación en actividades físicas de personas con y sin discapacidad. Revista Internacional de Ciencias del Deporte, 2(2), 49-64. Recuperado de http://www.cafyd.com/REVISTA/art4n2a06.pdf

Gutiérrez, M., y López, E. (2012). Clima motivacional, razones para la disciplina y comportamiento en Educación Física. Revista Internacional de Medicina y Ciencias de la Actividad Física y el Deporte, 12 (46), 235-251. Recuperado de http://cdeporte.rediris.es/revista/revista46/artclima292.pdf

Lagardera, F. (1989). La motivación en las clases de educación física. Apunts. Educación Física y deporte, (16-17), 105-108. Recuperado de http://www.revistaapunts.com/es/hemeroteca?article $=1104$ \&highlight=motivacion

McDonough, M. H., y Crocker, P. R. E. (2007). Testing self-determined motivation as a mediator of the relationship between psychological needs and affective and behavioral outcomes. Journal of Sport \& Exercise Psychology, 29(5), 645-663.

Méndez-Giménez, A., Fernández-Río, J., y Cecchini-Estrada, J. A. (2013). Climas motivacionales, necesidades, motivación y resultados en Educación Física. Aula Abierta, 41(1), 63-72.

Moreno, J. A., y Cervelló, E. (2004). Pensamiento del alumno hacia la Educación Física: su relación con la práctica deportiva y el carácter del educador. Enseñanza, 21, 345-362. Recuperado de http://espacio.uned.es/fez/ eserv.php?pid=bibliuned:20350\&dsID=pensamiento alumno.pdf 
Moreno, J. A., y Cervelló, E. (2010). La motivación autodeterminada en la actividad física y el deporte: conceptualización. En González-Cutre, D., Martínez, A., Gómez, A., y Moreno, J. A. (Coords.), Motivación en la actividad física y el deporte. (pp. 119-150). Sevilla: Wanceulen.

Moreno, J. A., González-Cutre, D., y Chillón, M. (2009). Preliminary validation in Spanish of a scale designed to measure motivation in physical education classes: the Perceived Locus of Causality (PLOC) Scale. The Spanish Journal of Psychology, 12, 327-337.

Moreno-Murcia, J. A., Joseph, P., y Huéscar, E. (2013). Cómo aumentar la motivación intrínseca en clases de educación física. E-motion. Revista de Educación, Motricidad e Investigación, 1, 30-39. Recuperado de http://www.uhu.es/ publicaciones/ojs/index.php/e-moti-on/article/view/2263/2156

Naranjo, M. L. (2009). Motivación: perspectivas teóricas y algunas consideraciones de su importancia en el ámbito educativo. Revista Educación, 33 (2), 153-170. Recuperado de http://www.revistas.ucr.ac.cr/index.php/educacion/article/view/ $\underline{510 / 525}$

Navarrete, B. (2009). La motivación en el aula. Funciones del profesor para mejorar la motivación en el aprendizaje. Innovación y Experiencias Educativas, 15, 1-9. Recuperado de http://www.csi-csif.es/andalucia/modules/mod ense/revista/pdf/ Numero 15/BELEN NAVARRETE 1.pdf

Navarro-Patón, R., Rodríguez, J. E., y Eírin, R. (2016). Análisis de la satisfacción de las necesidades psicológicas básicas, motivación y disfrute en Educación Física en Primaria. $\quad$ Sportis,2(3), 439-455. http://dx.doi.org/10.17979/sportis.2016.2.3.1758

Ntoumanis, N. (2001). A self-determination approach to the understanding of motivation in physical education. British Journal of Educational Psychology, 71(2), 225-242. doi: 10.1348/000709901158497

Ntoumanis, N., y Standage, M. (2009). Motivation in physical education classes: A selfdetermination theory perspective. Theory and Research in Education, 7(2), 194202. doi: $10.1177 / 1477878509104324$

Ryan, R. M., y Deci, E. L. (2000). Self-determination theory and the facilitation of intrinsic motivation, social development and well-being. American Psychologist, 55, 68-78.

Ryan, R. M., y Deci, E. L. (2017). Self-determination Theory: Basic Psychological Needs in Motivation, Development, and Wellness. New York: Guilford Press.

Sevil, J., Julián, J., Abarca-Sos, A., Aibar, A., y García-González, L. (2014). Efecto de una intervención docente para la mejora de variables motivacionales situacionales en Educación Física. Retos, 26, 108-113.

Valenzuela, J., y Nieto, A. M. (2008). Motivación y pensamiento crítico: Aportes para el estudio de esta relación. Revista Electrónica de Motivación y Emoción, 11 (28), 1-8. Recuperado de http://reme.uji.es/articulos/numero28/article3/article3.pdf

Valero, A., Delgado, M., y Conde, J. L. (2009). Motivación hacia la práctica del atletismo en la educación primaria en función de dos propuestas de enseñanza/aprendizaje. Revista de Psicología del Deporte, 18(2), 123-136. 


\section{ANEXOS}

\section{Anexo 1. Modelo de entrevista para los docentes}

$*$ DATOS DESCRIPTIVOS

\section{DATOS:}

- ¿Cuántos años tienes?

- ¿Cuándo terminaste tu formación universitaria inicial?

- ¿Cuántos años de experiencia como docente tienes?

- ¿En qué centro escolar trabajas? ¿A qué cursos impartes clases de EF?

\section{FORMACIÓN:}

- ¿Qué formación académica tienes?

- ¿Te sigues formando actualmente? ¿De qué manera?

- ¿Has recibido alguna formación relacionada con la motivación?

\section{* IDEOLOGía}

\section{CONCEPTO:}

- ¿Qué entiendes por motivación?

- ¿Qué tipos de motivación conoces? ¿Cuál crees que es más importante? ¿Por qué?

UTILIDAD:

- ¿Considera importante que el alumnado esté motivado en clases de EF?

- ¿Crees que es útil conocer y saber aplicar estrategias para motivar al alumnado?

\section{* DOCENCIA}

\section{DESARROLLO:}

- ¿De qué manera mantienes la motivación en el alumnado en el desarrollo de la clase?

- ¿Qué tipo de feedback o conocimiento de resultados sueles emplear con más frecuencia en clase? ¿Puedes ponerme un ejemplo?

- Tus alumnos de primer ciclo, ¿son capaces de hacer bien los juegos o ejercicios que planteas en clases? Coméntame que estrategias empleas para que se sientan competentes.

- ¿Cómo son las relaciones entre los/as niños/as en clases de EF? ¿Y con su maestro?

- ¿Haces algo en concreto para que esa relación sea buena, fluida, etc.? Puede ponerme algún ejemplo.

- En primer ciclo, ¿cómo describiría la autonomía de su alumnado? ¿Cómo trabaja la autonomía? Puede ponerme un ejemplo.

- ¿Le permite elegir algún juego o actividad, o valora la opinión y gustos de su alumnado con respecto a los juegos que se emplean en clases de EF?

- ¿Emplea el aprendizaje cooperativo con el alumnado de esa edad?

\section{* CONTEXTO}

\section{CONOCIMIENTO DE LA MOTIVACIÓN DE LOS ALUMNOS:}

- En general, ¿cómo describiría la motivación hacia las clases de EF del alumnado de primer ciclo?

\section{CAUSAS DE LA MOTIVACIÓN:}

- Por su experiencia o en base a su opinión, ¿cambia esa motivación hacia la asignatura de EF a lo largo de la educación obligatoria? ¿Por qué cree que ocurre?

\section{CONSECUENCIAS DE LA MOTIVACIÓN:}

- ¿Cuáles son las consecuencias de la motivación experimentada en las clases de EF? (Ya sea por estar muy motivado o tener una alta motivación o por todo lo contrario, por estar desmotivado)

\section{* SOLUCIONES}

PROPUESTAS: 
- ¿Podrías decirme algunas propuestas para mejorar la motivación del alumnado en clases de EF?

- ¿Qué juegos, actividades o contenidos son los que más le gustan al alumnado de primer ciclo?

- Por último, piense un momento, ¿hay alguna actividad o algo que haga en clases con lo que haya notado que el alumnado se entusiasma y disfruto mucho?

Muchas gracias por tu ayuda.

Anexo 2. Modelo de entrevista para los discentes

- ¿Cuántos años tienes?

- ¿Cuál es tu colegio?

\section{UTILIDAD:}

\section{* IDEOLOGIA}

- ¿Para qué crees tú que sirve hacer EF?

- ¿Crees que es bueno o malo hacer EF? ¿Por qué?

DIVERSIÓN:

- ¿Te diviertes en las clases de EF? ¿Por qué?

\section{* DOCENCIA}

\section{CONCEPCIONES SOBRE EL DOCENTE:}

- ¿Te gustan las clases que da tu maestro/a de EF?

- ¿Qué es lo que más gusta de él/ella?

- ¿Qué es lo que menos te gusta de él/ella?

DESARROLLO:

- Cuando estáis haciendo una actividad, un juego, etc. ¿El maestro/a te dice si lo estás haciendo bien? ¿Cómo te lo dice? ¿Cómo te sientes cuando te lo dice?

- Por el contrario, cuando estáis haciendo una actividad, un juego, etc. ¿El maestro/a te dice si lo estás haciendo mal? ¿Cómo te lo dice? ¿Cómo te sientes cuando te lo dice?

- En el desarrollo de la clase de EF, ¿participas en todas las actividades? ¿Por qué?

- ¿Eres capaz de hacer bien los ejercicios o los juegos que propone tu maestro?

- ¿Cómo te llevas con tus compañeros de clase? ¿Tienes muchos amigos? ¿Te portas bien con ellos? ¿Y ellos contigo (se portan o te tratan bien)? ¿Y con el maestro?

- ¿El maestro tiene en cuenta tu opinión? ¿Puedes elegir alguna vez algo en las clases de EF (con qué equipo juego, qué juego hacemos, etc.)? ¿Te gustaría poder elegir algún juego de los que hacéis en clases de EF?

\section{* JUEgos}

\section{CAUSAS DE LA MOTIVACIÓN/DESMOTIVACIÓN:}

- ¿Cuáles son los juegos que más te gustan en EF?

- ¿Cuáles son los juegos que no te gustan en EF?

CONSECUENCIAS DE LA DESMOTIVACIÓN:

- ¿Qué haces si no te gusta un juego? ¿Cómo te comportas?

\section{* SOLUCIONES}

\section{PROPUESTAS:}

- Imagínate que tú eres maestro/a de EF. ¿Qué juegos les harías a tus alumnos/as? Muchas gracias por tu ayuda. 